\title{
ISOTROPIC TRANSPORT PROCESS ON A RIEMANNIAN MANIFOLD
}

\author{
BY \\ MARK A. PINSKY( $\left.{ }^{(}\right)$
}

\begin{abstract}
We construct a canonical Markov process on the tangent bundle of a complete Riemannian manifold, which generalizes the isotropic scattering transport process on Euclidean space. By inserting a small parameter it is proved that the transition semigroup converges to the Brownian motion semigroup provided that the latter preserves the class $C_{0}$. The special case of a manifold of negative curvature is considered as an illustration.
\end{abstract}

1. Introduction. In order to construct a diffusion process on a differentiable manifold it is necessary, in general, to solve stochastic differential equations in coordinate patches and then piece together the resulting local diffusions [4]. In the case of the sphere $S^{n-1}$, Stroock [10] has shown that the Brownian motion may be obtained by solving a single stochastic differential equation on $R^{n}$, whose solution stays on the sphere of its own accord.

The purpose of this paper is to show that, on a wide class of Riemannian manifolds, the Brownian motion can be approximated in law by a globally defined stochastic process-the isotropic transport process. The paths of this process are piecewise geodesic. The joint position-velocity motion is a Markov process on the tangent bundle of the manifold. In the case of $R^{n}$, it coincides with the usual transport process [11]. Using a theorem of Kurtz [8], we show that the Brownian motion on the manifold can be approximated, in the sense of weak convergence, by a sequence of isotropic transport processes. As a by-product we obtain a formula for the Laplace-Beltrami operator as the spherical average of the second covariant derivative along the geodesic flow field in the tangent bundle.

2. Geodesic flow field. Let $M$ be a complete $C^{\infty}$ Riemannian manifold of dimension $n . T_{x}(M)$ denotes the tangent space at $x \in M ; T(M)$ denotes the tangent bundle over $M$. For $\tilde{x}=(x, \xi) \in T(M)$, we consider the geodesic flow $X=$ $\left\{X^{(x, \xi)}(t), t \geqslant 0\right\}$. This satisfies the properties that $X^{(x, \xi)}(0)=x, \dot{X}^{(x, \xi)}(0)=\xi$ and $\dot{X}^{(x, \xi)}(t)$ is a parallel vector field along $\left\{X^{(x, \xi)}(t), t \geqslant 0\right\}$.

Received by the editors May 11, 1975.

AMS (MOS) subject classifications (1970). Primary 60J25, 60J65, 53C20; Secondary 58G99, 47D05.

Key words and phrases. Isotropic transport process, geodesic flow, Brownian motion, contraction semigroup.

(1) Supported by the National Science Foundation MPS71-02838 A04. 
We consider the lifting of $X$ to a curve $\widetilde{X}$ in $T(M)$, defined by $\widetilde{X}^{(x, \xi)}(t)=$ $\left(X^{(x, \xi)}(t), \dot{X}^{(x, \xi)}(t)\right)$. Let $Z$ be the tangent vector to this curve at $t=0$. $Z$ is a vector field on $T(M)$ and is called the geodesic flow field $[6,132]$. We let $D_{Z}$ denote the operation of (covariant) differentiation defined by $Z$.

Let $C_{0}(T(M))$ be the Banach space of continuous functions on $T(M)$ with $\lim _{d\left(x, x_{0}\right) \rightarrow \infty} f(x)=0$. [Here $d$ is a metric consistent with the manifold topology and $x_{0}$ is a fixed point of $\left.M.\right] \quad C_{0}^{k}(T(M))=\left\{f \in C_{0}(T(M)): f\right.$ has $k$ continuous partial derivatives\}. $\|f\|=\sup _{\tilde{x} \in T(M)}|f(\tilde{x})|$. Finally, set

$$
\begin{array}{ll}
\left(T_{t}^{0} f\right)(\tilde{x})=f\left(\tilde{X}^{\tilde{x}}(t)\right), & (t>0) \\
\left(R_{\lambda}^{0} f\right)(\tilde{x})=\int_{0}^{\infty} e^{-\lambda t}\left(T_{t}^{0} f\right)(\tilde{x}) d t & (\lambda>0) .
\end{array}
$$

Proposition 2.1. $R_{\lambda}^{0}$ maps $C_{0}^{1}(T(M))$ into $C_{0}^{1}(T(M))$ and satisfies

$$
\left(\lambda-D_{Z}\right) R_{\lambda}^{0} f=f \quad\left(f \in C_{0}^{1}(T(M))\right) .
$$

ProOF. Let $f \in C_{0}^{1}(T(M))$. By the smooth dependence on initial conditions, $(x, \xi) \rightarrow \tilde{X}^{(x, \xi)}(t)$ is a $C^{\infty}$ mapping for each $t>0$. Therefore $T_{t}^{0} f \in$ $C^{1}(T(M))$. The dominated convergence theorem implies that $R_{\lambda}^{0} f \in C^{1}(T(M))$. To show that $R_{\lambda}^{0} f$ vanishes at $\infty$, let $\epsilon>0$. Choose $T>0$ such that $e^{-\lambda T}\|f\| / \lambda$ $\leqslant \epsilon / 2$. Since $f \in C_{0}(T(M))$, we can find $R>0$ such that $|f(\tilde{x})| \leqslant \epsilon \lambda / 2$ if $d\left(\tilde{x}, \tilde{x}_{0}\right) \geqslant R$. Now let $d\left(\tilde{x}, \tilde{x}_{0}\right) \geqslant R+T$; then $d\left(\tilde{X}_{t}, \tilde{x}_{0}\right) \geqslant R$ for $t \leqslant T$. Hence

$$
\begin{aligned}
\left|R_{\lambda}^{0} f(x)\right| & \leqslant\left|\int_{0}^{T} e^{-\lambda t} f(\widetilde{X}(t)) d t\right|+\left|\int_{T}^{\infty} e^{-\lambda t} f(\widetilde{X}(t)) d t\right| \\
& \leqslant \frac{\epsilon \lambda}{2}\left(\frac{1-e^{-\lambda T}}{\lambda}\right)+\frac{\epsilon}{2} \leqslant \epsilon,
\end{aligned}
$$

for $d\left(\tilde{x}, \tilde{x}_{0}\right) \geqslant R+T$. Thus $R_{\lambda}^{0} f \in C_{0}(T(M))$.

To verify (2.2) write, for $h>0$,

$$
\begin{aligned}
T_{h}^{0} R_{\lambda}^{0} f & =\int_{0}^{\infty} e^{-\lambda t} T_{t+h}^{0} f d t=e^{\lambda h} \int_{h}^{\infty} e^{-\lambda u} T_{u}^{0} f d u \\
& =e^{\lambda h}\left[R_{\lambda}^{0} f-\int_{0}^{h} e^{-\lambda u}\left(T_{u}^{0} f\right) d u\right]
\end{aligned}
$$

But $\left(T_{u}^{0} g\right)(\tilde{x})=g\left(\tilde{X}^{\tilde{x}}(u)\right)$. Therefore

$$
\begin{aligned}
D_{Z}\left(R_{\lambda}^{0} f\right) & \equiv \lim _{h \rightarrow 0} \frac{T_{h}^{0}\left(R_{\lambda}^{0} f\right)-R_{\lambda}^{0} f}{h} \\
& =\lim _{h \rightarrow 0}\left[\frac{e^{\lambda h}-1}{h} R_{\lambda}^{0} f-\frac{1}{h} \int_{0}^{h} e^{-\lambda u}\left(T_{u}^{0} f\right) d u\right]=\lambda R_{\lambda}^{0} f-f .
\end{aligned}
$$


3. Isotropic transport process. Let $(\Omega, B, P)$ be a probability space. On $\Omega$ we assume given a sequence $\left\{e_{n}\right\}_{n \geqslant 1}$ of independent random variables with the common distribution

$$
P\left\{e_{n}>t\right\}=e^{-t} \quad(t>0, n=1,2, \ldots) .
$$

We set $\tau_{0}=0, \tau_{n}=e_{1}+\cdots+e_{n}(n \geqslant 1)$. Let $\mu_{x}(d \eta)$ be the unique rotationally invariant probability measure on the unit sphere in $T_{x}(M)$. For $(x, \xi) \in$ $T(M)$, we define a sequence of random variables $\left\{Y_{1}, \xi_{1}, Y_{2}, \xi_{2}, \ldots\right\}$ as follows:

$$
Y_{0}=x, \quad \xi_{0}=\xi \text {. }
$$

Assuming that the joint distributions of $\left\{Y_{1}, \xi_{1}, \ldots, Y_{n-1}, \xi_{n-1}\right\}$ have been defined, we set

$$
\begin{array}{ll}
Y_{n}=X^{\left(Y_{n-1}, \xi_{n-1}\right)}\left(e_{n}\right) & (n=1,2,3, \ldots), \\
P\left\{\xi_{n} \in d \eta\right\}=\mu_{Y_{n}}(d \eta) & (n=1,2,3, \ldots) .
\end{array}
$$

It is readily verified that the probability law of $\left(Y_{1}, \xi_{1}, \ldots\right)$ is well defined by these conditional distributions. We now define a stochastic process $\{Y(t), t \geqslant 0\}$ by

$$
Y^{(x, \xi)}(t)=X^{\left(Y_{n}, \xi_{n}\right)}\left(t-\tau_{n}\right) \quad\left(\tau_{n} \leqslant t \leqslant \tau_{n+1}\right) .
$$

The curve $t \rightarrow Y(t) \in M$ is piecewise differentiable and therefore the tangent vector $\dot{Y}(t)$ is defined for $t \neq \tau_{n}(n \geqslant 1)$. We normalize $\dot{Y}(t)$ so that $\dot{Y}\left(\tau_{n}\right)=$ $\dot{Y}\left(\tau_{n}+0\right)$. The pair $\widetilde{Y}(t)=(Y(t), \dot{Y}(t))$ will be called the isotropic transport process on $T(M)$.

Let

$$
\begin{aligned}
& T_{t} f(\tilde{x})=E\left\{f\left(Y^{(x, \xi)}(t), \dot{Y}^{(x, \xi)}(t)\right)\right\} \\
& R_{\lambda} f(\tilde{x})=\int_{0}^{\infty} e^{-\lambda t} T_{t} f(\tilde{x}) d t .
\end{aligned}
$$

THEOREM 3.1. $\left\{T_{t}, t \geqslant 0\right\}$ is a strongly continuous contraction semigroup on $C_{0}(T(M))$. For $f \in C_{0}^{1}(T(M))$, the function $u=T_{t} f$ satisfies the differential equation

$$
\frac{\partial u}{\partial t}(t, x, \xi)=\left(D_{Z} u\right)(t, x, \xi)+\int_{T_{x}(M)}[u(t, x, \eta)-u(t, x, \xi)] \mu_{x}(d \eta) .
$$

This result will be established by working with the operators $\left\{R_{\lambda}, \lambda>0\right\}$. Of course the semigroup property follows from the Markov property which can be established along the lines of [2, pp. 65-68]. But since we make no explicit use of the Markov property below, this proof is omitted.

Proposition 3.2. $R_{\lambda}$ maps $C_{0}(T(M))$ into $C_{0}(T(M))$ and satisfies

$$
R_{\lambda} f=R_{1+\lambda}^{0} f+R_{1+\lambda}^{0} P R_{\lambda} f \quad\left(f \in C_{0}(T(M))\right)
$$

where $P f(x, \xi)=\int_{T_{x}(M)} f(x, \eta) \mu_{x}(d \eta)$. 
Proof. Following a similar argument above, we have

$$
\left(R_{\lambda} f\right)(\tilde{x})=\left\{\int_{0}^{T}+\int_{T}^{\infty}\right\} e^{-\lambda t} E f(\tilde{Y}(t)) d t .
$$

Given $\epsilon>0$, choose $T$ such that $e^{-\lambda T}\|f\| / T<\epsilon / 2$. Choose $R$ such that $|f(\tilde{x})| \leqslant$ $\epsilon$ for $d\left(x, x_{0}\right) \geqslant R$. Then for $d\left(x, x_{0}\right) \geqslant R+T$, the first integral is $\leqslant \epsilon / 2$. Thus $\left|R_{\lambda} f(\tilde{x})\right| \leqslant \epsilon$ for $d\left(x, x_{0}\right) \geqslant R+T$. To prove (3.2a), we write

$$
\begin{aligned}
\left(R_{\lambda} f\right)(\tilde{x})= & E\left\{\int_{0}^{\tau_{1}}+\int_{\tau_{1}}^{\infty}\right\} e^{-\lambda t} f(\tilde{Y}(t)) d t \\
= & E \int_{0}^{\infty} I_{\left(t<\tau_{1}\right)} e^{-\lambda t} f(\tilde{X}(t)) d t \\
& +E\left\{e^{-\lambda \tau_{1}} \int_{0}^{\infty} e^{-\lambda s} f\left(\tilde{X}\left(\tau_{1}+s\right)\right) d s\right\} .
\end{aligned}
$$

The first integral $=\int_{0}^{\infty} e^{-t} e^{-\lambda t} f(\widetilde{X}(t)) d t=\left(R_{1+\lambda}^{0} f(\tilde{x})\right.$. For the second, note that $\widetilde{X}\left(\tau_{1}+s\right)=\left(X^{\left(Y_{1}, \xi_{1}\right)}(s), X^{\left(Y_{1}, \xi_{1}\right)}(s)\right)$. Taking the conditional expectation with respect to $\left(Y_{1}, \xi_{1}\right)$ and noting that $Y_{1}=X^{(x, \xi)}\left(\tau_{1}\right)$, we have

$$
\begin{aligned}
E\left\{e^{-\lambda \tau_{1}} \int_{0}^{\infty} e^{-\lambda s} f\right. & \left.\left(\tilde{X}\left(\tau_{1}+s\right)\right) d s\right\}=E\left\{e^{-\lambda \tau_{1}}\left(R_{\lambda} f\right)\left(Y_{1}, \xi_{1}\right)\right\} \\
& =E\left\{e^{-\lambda \tau_{1}}\left(P R_{\lambda} f\right)\left(Y_{1}\right)\right\}=\int_{0}^{\infty} e^{-\lambda s}\left(P R_{\lambda} f\right)\left(X^{(x, \xi)}(s)\right) e^{-s} d s \\
& =\left(R_{1+\lambda}^{0} P R_{\lambda} f\right)(x, \xi) .
\end{aligned}
$$

Proposition 3.3. Let $f \in C_{0}^{1}(T(M))$. Then $R_{\lambda} f \in C_{0}^{1}(T(M))$ and

$$
\left(\lambda-D_{Z}-P+I\right)\left(R_{\lambda} f\right)=f .
$$

Proof. Using Proposition 3.2, we iterate (3.2a), obtaining

$$
R_{\lambda} f=R_{1+\lambda}^{0} f+\sum_{n=1}^{\infty}\left(R_{1+\lambda}^{0} P\right)^{n}\left(R_{1+\lambda}^{0} f\right) .
$$

The series converges uniformly due to the estimation $\left\|R_{1+\lambda}^{0} P\right\| \leqslant 1 /(1+\lambda)$. Thus $R_{\lambda} f \in C_{0}(T(M))$. For $f \in C_{0}^{1}(T(M))$, this series may be differentiated termby-term and the differentiated series also converges uniformly. Hence $R_{\lambda} f \in$ $C_{0}^{1}(T(M))$ which was to be shown.

To prove (3.3a), we apply the operator $\left(1+\lambda-D_{Z}\right)$ to both sides of (3.2a) and use (2.2). Thus

$$
\left(1+\lambda-D_{Z}\right) R_{\lambda} f=f+P R_{\lambda} f
$$

which was to be proved. 
Proposition 3.4. Let $u \in C_{0}^{1}(T(M)),\left(\lambda-D_{Z}-P+D\right) u=0$ for some $\lambda>0$. Then $u \equiv 0$.

Proof. Assume that $\sup _{\tilde{x} \in T(M)} u(x)>0$. Then this sup is assumed at some $\tilde{x}_{0} \in M$, for otherwise $3 \tilde{x}_{n} \rightarrow \infty$ such that $u\left(\tilde{x}_{n}\right) \rightarrow \sup u(x)>0$ which contradicts $f \in C_{0}(T(M))$. Now at $\tilde{x}_{0}, D_{z} u\left(\tilde{x}_{0}\right)=0$ and $P u\left(\tilde{x}_{0}\right)-u\left(\tilde{x}_{0}\right) \leqslant 0$. But $P u\left(\tilde{x}_{0}\right)-u\left(\tilde{x}_{0}\right)=\lambda u\left(\tilde{x}_{0}\right)>0$, a contradiction. Therefore $u(\tilde{x}) \leqslant 0$ on $T(M)$. Applying the argument to $-u$, we see that $u(\tilde{x}) \geqslant 0$ on $T(M)$. Thus $u \equiv 0$.

To complete the proof of Theorem 3.1, we appeal to the Hille-Yosida theorem. $u=R_{\lambda} f$ is the unique solution of the equation $\left(\lambda-D_{Z}-P+D u=f\right.$ and satisfies $\|u\| \leqslant\|f\| / \lambda$. Hence there exists a strongly continuous contraction semigroup $\left\{\bar{T}_{t}, t \geqslant 0\right\}$ whose resolvent operators are given by $\left\{R_{\lambda}, \lambda>0\right\}$. By the uniqueness of Laplace transform, we conclude that $\bar{T}_{t}=T_{t}$.

To identify the domain of $T_{t}$, we recall that $D=R_{\lambda}\left(C_{0}(T(M))\right)$ which is independent of $\lambda$. Clearly $C_{0}^{1}(T(M)) \subseteq D$. Now if $u \in D, u=R_{\lambda} f$ for some $f \in$ $C_{0}(T(M))$. By 3.2a, $u=R_{1+\lambda}^{0} g, g=f+P R_{\lambda} f$. But for any $g \in C_{0}(T(M))$ the proof of Proposition 2.1 shows that $R_{\lambda}^{0} g$ is differentiable in the $Z$ direction, and that $D_{Z}\left(R_{\lambda}^{0} g\right)=\lambda\left(R_{\lambda}^{0} g\right)-g$. Hence $D_{Z} u$ exists and is an element of $C_{0}(T(M))$. Thus $D=\left\{u \in C_{0}(M): D_{z} u \in C_{0}(M)\right\}$. The proof is now complete.

4. Convergence to Brownian motion. We introduce a parameter $\epsilon>0$ and consider a one-parameter family of isotropic transport processes corresponding to the backward equation

$$
\partial u / \partial t=\epsilon D_{z} u+(P u-u) .
$$

This process can be constructed by replacing $\left\{\xi_{n}\right\}_{n \geq 1}$ by $\left\{\epsilon \xi_{n}\right\}_{n \geq 1}$ in the definition of $\widetilde{Y}(t)$. The solution of equation (4.1) with $u(0, x, \xi)=f(x, \xi)$ will be denoted by $T_{t}^{(\epsilon)} f$.

The Laplace-Beltrami operator $\Delta$ defines a Markov process on $M$, the Brownian notion $\left\{B^{x}(t), t<\zeta\right\}$ where $\zeta$ is the lifetime. We introduce the semigroup

$$
U_{t} f(x)=E\left\{f\left(B^{x}(t)\right), t<\zeta\right\}
$$

whose infinitesimal generator is an extension of $\Delta$. We shall assume the following:

$$
U_{t} \text { maps } C_{0}(T(M)) \text { into } C_{0}(T(M)) \text {. }
$$

THEOREM 4.3. Assume (4.2). Then for $f \in C_{0}(M)$,

$$
\lim _{\epsilon \rightarrow 0} T_{t / \epsilon^{2}}^{(\epsilon)} f=U_{t / n} f
$$

uniformly on $M$.

If (4.2) is satisfied, then $P(\zeta=\infty)=1$, but not conversely. (4.2) is satisfied if (a) $M$ is compact of (b) $M$ has bounded negative curvature (see below). 
To prepare the proof, we set $B=P-I, A=D_{Z}$. Then

$$
\begin{gathered}
P A P f=0 . \quad f \in C_{0}^{1}(T(M)) . \\
\{f: B f=0\}=C_{0}(M), \\
\lim _{t \rightarrow \infty} e^{t B} f=P f, \quad f \in C_{0}(T(M)) .
\end{gathered}
$$

The following proposition defines $B^{-1}$.

Proposition 4.7. Let $g \in C_{0}(T(M))$ with $\int_{T_{x}(M)} g(x, \xi) \mu_{x}(d \xi)=0$. The unique solution of the equations

$$
B f=g, \quad \int_{T_{x}(M)} f(x, \xi) \mu_{x}(d \xi)=0
$$

is given by $f(x, \xi)=-g(x, \xi)$.

The proof is omitted.

We now define the operator

$$
C=P A B^{-1} A P \text {. }
$$

Proposition 4.8. Let $f \in C_{0}^{2}(M)$. Then $C f=n^{-1} \Delta f$, where $\Delta$ is the Laplace-Beltrami operator on $M$.

PROOF. We first verify that the components of any covariant vector $\left(\xi_{1}\right)$ satisfy

$$
\int_{T_{x}(M)} \xi_{i} \xi_{j} \mu_{x}(d \xi)=g_{i j}(x) / n \quad(1 \leqslant i, j \leqslant n) .
$$

Indeed, by the rotational invariance of $\mu_{x}$, we must have $\int\langle\dot{\xi}, \mu \backslash \xi, \nu\rangle \mu_{x}(d \xi)=\langle\mu, \nu\rangle / n$ for any two vectors $\mu, v \in T_{x}(M)$. The left-hand member of this equation is $\mu_{k} \nu_{s} g^{i k}(x) g^{j s}(x) \int \xi_{i} \xi_{j} \mu_{x}(d \xi)$ and the right-hand side is $g^{i j}(x) \mu_{i} \nu_{j}$. Clearly the only possible choice is (4.9). Now we write the second covariant derivative

Using (4.7a), we have

$$
D_{Z} D_{Z} f=g^{i l} \xi_{l} g^{j s} \xi_{s}\left(\frac{\partial^{2} f}{\partial x^{i} \partial x^{j}}-\Gamma_{i j}^{k} \frac{\partial f}{\partial x^{k}}\right)
$$

$$
\begin{aligned}
n P D_{Z} D_{Z} f & =g_{l s}(x) g^{i l}(x) g^{j s}(x)\left(\frac{\partial^{2} f}{\partial x^{i} \partial x^{j}}-\Gamma_{i j}^{k} \frac{\partial f}{\partial x^{k}}\right) \\
& =g^{i j}(x)\left(\frac{\partial^{2} f}{\partial x^{i} \partial x^{i}}-\Gamma_{i j}^{k} \frac{\partial f}{\partial x^{2}}\right) \\
& =g^{i j}(x) D_{i} D_{j} f,
\end{aligned}
$$

which is the usual expression for the Laplace-Beltrami operator [5].

Proof of Theorem 4.3. We apply the method of T. G. Kurtz [8, Theorem 2.2]. $u=T_{t / \epsilon}^{(\epsilon)} f$ satisfies the equation 


$$
d u / d t=A / \epsilon+B u / \epsilon^{2}
$$

where

$$
P A P=0, \quad \lim _{\lambda \rightarrow \infty} \int_{0}^{\infty} \lambda e^{-\lambda t} e^{t B} f d t=P f
$$

We must show that $C_{0}^{1}(M) \subseteq$ the closure of the range of $\lambda-\Delta, \lambda>0$. But (4.2) implies [1] that the resolvent operator maps $C_{0}(M) \rightarrow C_{0}(M)$. Therefore, the equation $(\lambda-\Delta) f=g \in C_{0}^{1}(M)$ is solved by $f(x)=E\left\{\int_{0}^{\infty} e^{-\lambda t} g\left(B_{t}^{x}\right) d t\right\} \in C_{0}(M)$. Hence by the theorem of Kurtz, we have for $f \in C_{0}^{1}(M)$,

$$
\lim _{\epsilon \rightarrow 0} e^{t\left(A / \epsilon+B / \epsilon^{2}\right)} f=e^{t P A B^{-1} A P} P f=e^{t \Delta / n} P f
$$

in the norm of $C_{0}(T(M))$, which was to be shown.

5. Manifolds of negative curvature. In order to verify (4.2) in some noncompact cases, we assume in addition that $M$ is an analytic simply connected manifold of negative curvature. In this case, the problem was considered by Azencott [1], who used a method based on Hasminskii's test. We will show below that by using a simple observation of Itô [7], (4.2) may be proved by a direct examination of the stochastic equations.

Indeed, in this case $M$ is homeomorphic to its tangent space at some $x_{0} \in$ $M$. Taking geodesic polar coordinates at $x$, we have [3]

$$
r^{2}\left(B_{t}\right)=\int_{0}^{t} 2 r\left(B_{s}\right) d W_{s}+\int_{0}^{t}\left[2+2 \frac{\Theta^{\prime}}{\Theta}\left(B_{s}\right) r\left(B_{s}\right)\right] d s
$$

where $\Theta$ is the volume element in these coordinates $\Theta^{\prime} / \Theta \leqslant(n-1) b$ coth $b r$, where $K \geqslant-b^{2}$. Now we apply Itô's formula to $f=\log \left(1+r^{2}\right)$ :

$$
\begin{aligned}
\log \left[1+r^{2}\left(B_{t}\right)\right]= & \int_{0}^{t} \frac{2 r\left(B_{s}\right) d W_{s}}{1+r^{2}\left(B_{s}\right)}+\int_{0}^{t} \frac{2+2\left(\Theta^{\prime} / \Theta\right)\left(B_{s}\right) r\left(B_{s}\right)}{1+r^{2}\left(B_{s}\right)} d s \\
& -\int_{0}^{t} \frac{4 r^{2}\left(B_{s}\right)}{\left[1+r^{2}\left(B_{s}\right)\right]^{2}} d s .
\end{aligned}
$$

To estimate the expectation, note that the first term above is an $L^{2}$-martingale and hence has mean 0 . The third term is negative. For the second term, note that $|x \operatorname{coth} x| \leqslant 2+2 x$. Therefore

$$
\begin{aligned}
E\left\{\log \left[1+r^{2}\left(B_{t}\right)\right]\right\} & \leqslant \int_{0}^{t} \frac{2+4(n-1)\left[1+b r\left(B_{s}\right)\right]}{1+r^{2}\left(B_{s}\right)} d s \\
& \leqslant[4 n-2+2 b(n-1)] t
\end{aligned}
$$

where we have used the inequality $r /\left(1+r^{2}\right) \leqslant 1 / 2$.

Proposition 5.1. Let $M$ be an analytic simply connected Riemann mani- 
fold with $K \geqslant-b^{2}$. Then the diffusion semigroup $U_{t}$ maps $C_{0}(M)$ into $C_{0}(M)$.

Proof. Let $f \in C_{0}(M)$ : given $\epsilon>0$, let $K_{R}$ be a geodesic ball such that $|f(x)| \leqslant \epsilon$ if $x \notin K_{R}$. Now

$$
U_{t} f(x)=E f\left(B_{t}^{x}\right)=\left\{\int_{K_{R}^{c}}+\int_{K_{R}}\right\} f(y) P_{t}^{x}(d y) .
$$

The first integral is bounded by $\epsilon$, by definition of $K_{R}$. To estimate the second integral, notice that if $B_{t}^{x} \in K_{R}$, then $d\left(B_{t}^{x}, x\right) \geqslant d\left(x, x_{0}\right)-R$. Therefore if $d\left(x, x_{0}\right)>R$,

$$
\begin{aligned}
\int_{K_{R}^{c}} P_{t}^{x}(d y) & \leqslant P\left\{d\left(B_{t}^{x}, x\right) \geqslant d\left(x, x_{0}\right)-R\right\} \\
& \leqslant P\left\{\log \left[1+d^{2}\left(B_{t}^{x}, x\right)\right] \geqslant \log \left[1+\left(d\left(x, x_{0}\right)-R\right)^{2}\right]\right\} \\
& \leqslant t(4 n-2+2 b(n-1)) / \log \left(1+\left(d\left(x, x_{0}\right)-R\right)^{2}\right) .
\end{aligned}
$$

Now let $d\left(x, x_{0}\right) \rightarrow \infty$. Thus $\overline{\lim }\left|T_{t} f(x)\right| \leqslant \epsilon$ for each $\epsilon>0$. Hence $U_{t} f \in$ $C_{0}(M)$ which was to be proved.

ADDED IN PROOF. We have just learned of similar approximations of Brownian motion by geodesics in the works of Jorgensen [12] and Malliavin [13]. The latter construction generalizes the classical balayage method of Poincaré.

\section{REFERENCES}

1. R. Azencott, Behavior of diffusion semigroups at infinity, Bull. Soc. Math. France 102 (1974), 193-240.

2. R. M. Blumenthal and R. K. Getoor, Markov processes and potential theory, Pure and Appl. Math., vol. 29, Academic Press, New York and London, 1968. MR 41 \#348.

3. A. Debiard, B. Gaveau and E. Mazet, Temps d'arrêt des diffusions riemanniennes, C. R. Acad. Sci. Paris Sér. A.-B 278 (1974), A723-A725. MR 49 \#6381a.

4. R. Gangolli, On the construction of certain diffusions on a differentiable manifold, Z. Wahrscheinlichkeitstheorie und Verw. Gebiete 2 (1964), 406-419. MR 29 \#2870.

5. R. Goldberg, Curvature and homology, Academic Press, New York, 1968.

6. N. J. Hicks, Notes on differential geometry, Van Nostrand Math. Studies, no. 3, Van Nostrand, Princeton, N. J., 1965. MR 31 \#3936.

7. K. Itô, Stability of stochastic dynamical systems, Lecture Notes in Math., vol. 294, Springer-Vorlag, New York, 1973, pp. 1-7.

8. T. G. Kurtz, A limit theorem for perturbed operator semigroups with applications to random evolutions, J. Functional Analysis 12 (1973), 55-67.

Ann. Probability 3 (1975), 618-642.

10. D. W. Stroock, On the growth of stochastic integrals, 2 . Wahrscheinlichkeitstheorie und Verw. Gebiete 18 (1971), 340-344. MR 44 \#4825.

11. S. Watanabe and T. Watanabe, Convergence of isotropic scattering transport process to Brownian motion, Nagoya Math. J. 40 (1970), 161-171. MR 43 \#5606.

12. E. Jorgensen, The central limit problem for geodesic random walk, $\mathrm{Z}$. Wahrscheinlichkeitstheorie und Verw. Gebiete 32 (1975), 1-64.

13. P. Malliavin, Diffusions et géométrie différentielle globale, Lecture Notes, August 1975, Institut Henri Poincaré, 11 rue Pierre et Marie Curie, Paris 5.

DEPARTMENT OF MATHEMATICS, NORTHWESTERN UNIVERSITY, EVANSTON, ILLINOIS 60201 